\title{
МЕТОД ИНТЕГРИРОВАННОЙ ОЦЕНКИ УСТАЛОСТНЫХ НАПРЯЖЕНИЙ В СТРУКТУРЕ ВОССТАНОВЛЕННЫХ ЛОПАТОК ТЭЦ И ГЭС
}

\author{
Савинкин Виталий Владимирович', \\ cavinkin7@mail.ru
}

\author{
Кузнецова Виктория Николаевна², \\ dissovetsibadi@bk.ru
}

\author{
Ратушная Татьяна Юрьевна', \\ tratushnaia@mail.ru
}

\author{
Киселев Леонид Александрович ${ }^{3}$, \\ remplazma@mail.ru

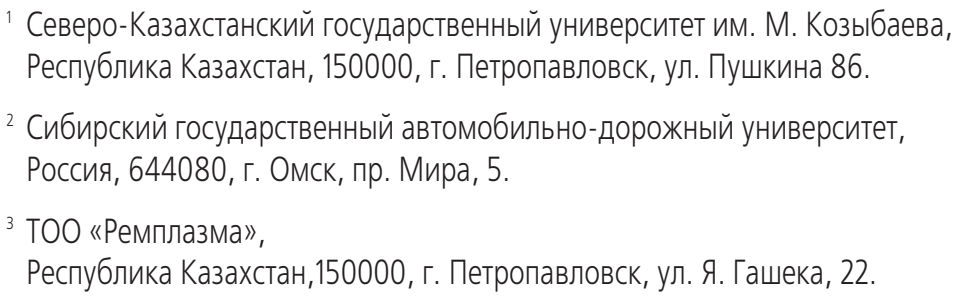

Актуальность исследования обусловлена необходимостью разработки методики точного определения ресурсной долговечности и надежности высоконагруженного оборудования, используемого для производства и преобразования энергии на основе георесурсов (ГЭС, ТЭЦ), основанной на выявлении зон концентрации внутренних напряжений как показателя фазовых изменений в структуре материала деталей.

Цель: повышение ресурсной долговечности нагруженных лопаток турбины ГЭС и ТЭЦ за счет внедрения разработанной интегрированной методики точного прогнозирования структурно-фазовых изменений на стадии зарождения усталостных дефектов. Объект: лопатки турбин ТЭЦ и ГЭС, восстановленные источником лазерно-плазменной энергии.

Применялся комплексный метод исследования концентрации внутренних напряжений в структуре нагруженных деталей, подверженных динамическим нагрузкам. Использовалась методология системного анализа, а также метод распределения поля остаточной намагниченности в деградированной микроструктуре материала. При разработке алгоритма интегрированного метода диагностики и точного прогнозирования применялись методы математической статистики. Восстановленные детали исследовались методами неразрушающего контроля с последующим анализом и обработкой полученных результатов.

Результаты. Разработан алгоритм проведения диагностики восстановленных деталей высокотехнологичных комплексов производства и преобразования энергии георесурсов на примере лопаток турбины ГЭС. Сформирована база данных концентраций напряжений лопатки в зависимости от фазовой структуры металла, позволяющая установить причинно-следственные связи между дефектом, технологическими режимами восстановления и режимами эксплуатации агрегата. Решена научная проблема по созданию единой физической модели, и определены границы применимости данного метода. Установлен безопасный режим эксплуатации энергетического оборудования $\left(H_{x}=62-76 \mathrm{~A} / \mathrm{M}\right)$, повышающий ресурс турбины в 1,7 раза.

\section{Ключевые слова:}

Усталостные напряжения, фазовая структура, лопатка турбины, интегрированная оценка, методы неразрушающего контроля

\section{Введение}

Экстремальные условия эксплуатации, дефицит технологий восстановления энергетического комплекса и машиностроительного оборудования Казахстана, России и других стран СНГ подтверждают, что на качество, безопасность и энергоэффективность производства и преобразования вырабатываемой энергии на основе георесурсов существенное влияние оказывает ресурс нагруженных турбоагрегатов. На сегодняшний день на территории СНГ работает более 500 тепло- и гидроэлектростанций. Доля морально устаревших турбин, по приблизительным подсчетам, составляет около $65 \%$, при этом темпы роста цен на энергетические ресурсы, техническое обслуживание катастрофически растут.
Для получения тепловой и электрической энергии в энергетике используют турбины с вращающимся рабочим органом ротора с закрепленными на нем лопатками, приводимыми в действие потоком давления пара, образованным в результате сгорания измельченного угля, или потоком воды. Таким образом, происходит преобразование одного вида энергии (давление пара, воды) в другой (тепло и электричество).

В ходе эксплуатации лопатки турбин подвергаются усиленному эрозионному изнашиванию [1]. Непредвиденные поломки оборудования ТЭЦ или ГЭС, сопряженные с выходом из строя гидротурбины, короблением, обрывом, эрозионным износом рабочих лопаток $n$-ступени и усталостным вибрационным разрушением $[2,3]$ влечет за собой техно- 
генные аварии и сбои в системе подачи электроэнергии в стране [4]. Электрическая и тепловая энергия задают главный вектор развития энергетической отрасли с возможностью эффективного использования георесурсов.

Неисправности на многих ГЭС и ТЭЦ выявили острую необходимость в методике точного прогнозирования ресурсной надежности, восстановления и мониторинга состояния энергетического оборудования, используемого для производства и преобразования энергии георесурсов, на структурно-фазовом уровне материала агрегата. Научно-обоснованный подход к оценке и диагностике усталостной надежности энергетического комплекса с учетом системных связей динамических нагрузок и структурных изменений в материале лопаток турбин в мировой практике энергетической отрасли не применяется и не имеет нормативного регулирования.

Исследование данных процессов позволит определять места высоконагруженных агрегатов, работающих на стадии зарождения внутренних скрытых дефектов, а не в момент их концентрации, как принято в классических расчетных методиках. Основные результаты исследований позволят не только идентифицировать зарождающийся дефект, но и интерпретировать его, что повысит надежность, долговечность и эффективность работы турбин ТЭЦ и ГЭС.

Для решения поставленной задачи необходима как модернизация методологического подхода, так и создание метрологического обеспечения точного прогнозирования структурно-фазового состояния и усталостной долговечности турбоагрегата, что позволит повысить качество неразрушающего контроля энергетического оборудования, долговечность и безопасность ГЭС и ТЭЦ.

Основными требованиями, предъявляемыми к лопаткам паровых и газовых турбин, является соответствие их проектной геометрии и размерных параметров требованиям чертежей, а также высокий уровень физико-механических свойств (прочность, твердость, сопротивления усталости, фреттинг-стойкость, эрозионной и коррозионной стойкости) [5].

Ресурсная долговечность и надежность деталей определяется их жизненным циклом. К основным дефектам, влияющим на долговечность нагружаемых лопаток, можно отнести следующие: износ, деформация, вырыв, изменение физико-механических свойств конструкционных элементов и накопление усталостных напряжений в лопатках турбины. Научные изыскания подтвердили, что первостепенная причина зарождения подобных дефектов в энергонагруженных агрегатах находится в локальных участках формирования усталостных напряжений (ЗКН - зоны концентрации напряжений). Это участки динамического и механического воздействия, где прогрессируют процессы коррозии, усталости, нарушая структурную связь кристаллической решетки материала. Следовательно, определение ЗКН оборудования, как показателя структурных изменений, является актуальной задачей диагностики и прогнозирования ресурса турбины.

Несмотря на значительное количество работ в области прогнозирования остаточного ресурса, создание научно-методической базы методик выполнения измерений и комплексной оценки мест локализации структурных дефектов на стадии их зарождения с целью повышения надежности изделий сложной формы при неразрушающем контроле представляется перспективной областью исследований.

Теоретическое обоснование экспериментального интегрированного метода контроля, идентификации

и интерпретации измерений напряженности восстановленной структуры

Основная идея работы заключается в исследовании концентрации внутренних напряжений в структуре нагруженных деталей на участках, подверженных максимальным динамическим нагрузкам, что позволит установить основные зависимости повышения надежности и эффективной эксплуатации. Исследуя процесс диагностики как отдельный этап жизненного цикла лопаток турбин и инструмент прогнозирования надежности и долговечности, определены основные методы неразрушающего контроля, получившие широкое применение в мировой практике машиностроения. К ним относятся ультразвуковой, акустико-эмиссионный, магнитопорошковый и другие. Классическая дефектоскопия обеспечивает обнаружение только несплошностей в металле и не позволяет использовать данные методы для оценки изменений свойств лопатки на структурно-фазовом уровне в момент зарождения процессов усталости в металле.

На сегодняшний день усовершенствован и успешно внедряется во многих сферах деятельности промышленных предприятий по преобразованию энергии георесурсов уникальный способ диагностики изделий, в основу которого положен комплекс известных физических явлений, таких как магнитоупругий эффект и рассеяние магнитного потока (MFL - Magnetic Flux Leakage) в слабых магнитных полях [6-8].

Данный метод обладает рядом преимуществ, его применение не требует специальных намагничивающих устройств; заранее не известные места концентрации напряжений от рабочих нагрузок определяются в процессе их контроля. Не требуется предварительная подготовка поверхности деталей. Средства измерений, реализующие данный метод, имеют малые габаритные размеры, автономное питание и совместимы с персональным компьютером, что позволяет автоматизировать обработку результатов измерений и контроля.

K основным недостаткам можно отнести низкую устойчивость к внешним воздействиям, но главная его проблема заключается в том, что не из- 
вестно, какая зона концентрации (рассеяния) напряженности остаточного магнитного поля (ОМП) соответствует зоне концентрации механических напряжений от дефектов физической несплошности металла лопатки $[9,10]$. Для решения этой проблемы необходим физический энергетический критерий оценки магнитного состояния. Такой критерий существует, но в рассматриваемом методе предложен и используется частный показатель (не критерий) математической производной напряженности ОМП. Напряженность принудительно наведенного магнитного поля и ОМП не коррелируют и не имеют прямой зависимости от деформированного состояния и структурно-фазового состава металла. Также одна из научных проблем заключается в отсутствии обоснованной степени воспроизводимости результатов измерительного эксперимента. В этом основной недостаток методологии анализа магнитограмм OMП в методе MFL.

В производственной практике принцип вихревых токов играет ключевую роль при контроле параметров в процессе проведения технической диагностики. Сущность вихретокового метода базируется на эффекте согласованного проявления внешнего электромагнитного поля и поля вихревых токов, создаваемых соленоидом катушки возбуждения в электропроводящей структуре лопатки. Электромагнитные характеристики и геометрические параметры исследуемой лопатки, а также координатное положение датчиков вихревых токов (ДВТ) оказывают значительное воздействие на плотность вихревых токов в исследуемом объекте. Следовательно, характеристики лопатки воздействуют на ЭДС датчика, т. е. результаты измерений содержат большее количество выходных параметров. Внешние факторы рабочей среды не снижают качество сигнала вихретокового датчика и достоверности результатов.

Важной научной проблемой данного метода является отсутствие единой строгой физической модели и установления границ применимости данного метода, что ставит под сомнение однозначность получаемых результатов предшествующих исследователей. Практика современного ремонтно-восстановительного производства показывает необходимость в универсальном методе оценки качественных и аппроксимирующих количественные показатели измеряемых параметров. Данная методика должна иметь метрологическое обеспечение с достоверной статистической базой.

Исследованиями ведущих ученых, таких как А.А. Дубов, Е.А. Демин, А.И. Миляев, О.И. Стеклов, B.M. Матюнин, R. Zoughi, M.J. Lovejoy, N. Diallo, S. Mamadou, N. Fromer, A. Neil, S. Myung $[11,12]$ и др., установлено, что при неразрушающем контроле изделий, кроме самой оценки мест локализации скрытых дефектов (микротрещин, питтинга, усталостных напряжений), важной задачей стоит точность измерений и установление причин зарождения очагов дефектов. Решить данную задачу можно путем синтеза совре- менных методов неразрушающего контроля и методики прогнозирования технического состояния объекта исследования.

Е.С. Гаркунов, М.Б. Аркулис, М.П. Барышников, Н.И. Мишенева, Ю.И. Савченко [13, 14] в своих работах утверждают, что применяемые методы неразрушающего контроля имеют направленность на обнаружение уже сформированных трещин и других скрытых дефектов, и они не позволяют осуществить раннюю диагностику структуры детали на стадии зарождения повреждений. Применение предложенного комплексного метода технической диагностики в сочетании с проверочными расчетами на прочность и вероятностным прогнозированием наиболее напряженных узлов турбины делает задачу определения реального ресурса лопаток турбины выполнимой.

Кроме того, предлагаемый способ диагностирования позволяет по характеристике распределения поля остаточной намагниченности $\mathrm{H}_{\mathrm{x}}$ вдоль поверхности детали, определить тон колебаний, т. е. оценить вибрационную надежность лопаток турбины. В этом случае используется әффект поглощения энергии механических колебаний (демпфирование колебаний), который вызывает соответствующий рост остаточной намагниченности лопаток.

Схема контроля восстановленных лопаток после эксплуатации по предлагаемому методу представлена на рис. 1.

Этап № 1. Паралетры и условия эксплуатаuии. На начальном этапе необходимо проанализировать условия эксплуатации, техническую документацию, а также факторы, влияющие на снижение надежности оборудования. Далее определить марку материала и его прочностные критерии, химикокомпонентый состав, сферу и особенности эксплуатации, цикличность работы и расчетные нагрузки.

Этап № 2. Оперативно-технологические излерения исследуелой лопатки. Регистрация режимов работы, параметров, характеризующих конструктивно-технологические особенности, установление зависимости параметров технологического цикла, таких как: номинальное давление, рабочая температура, вибрации от воздействия рабочей среды. После регистрации вышеперечисленных параметров проводится их сопоставление со справочными и расчетными данными для внесения поправок в информационный банк данных.

Этап № 3. Критическая оченка результатов. Формирование максимально объективной информации о механических свойствах структуры лопаток. Реализуется определение и классификация дефектов, установление причинно-следственных связей и последовательность процессов их возникновения и развития. Данный шаг является значимым, так как задействованы значительные объемы средств измерений и методов обнаружения скрытых дефектов. Одновременно формируется информационный резерв данных для прогнозирования ресурса. 


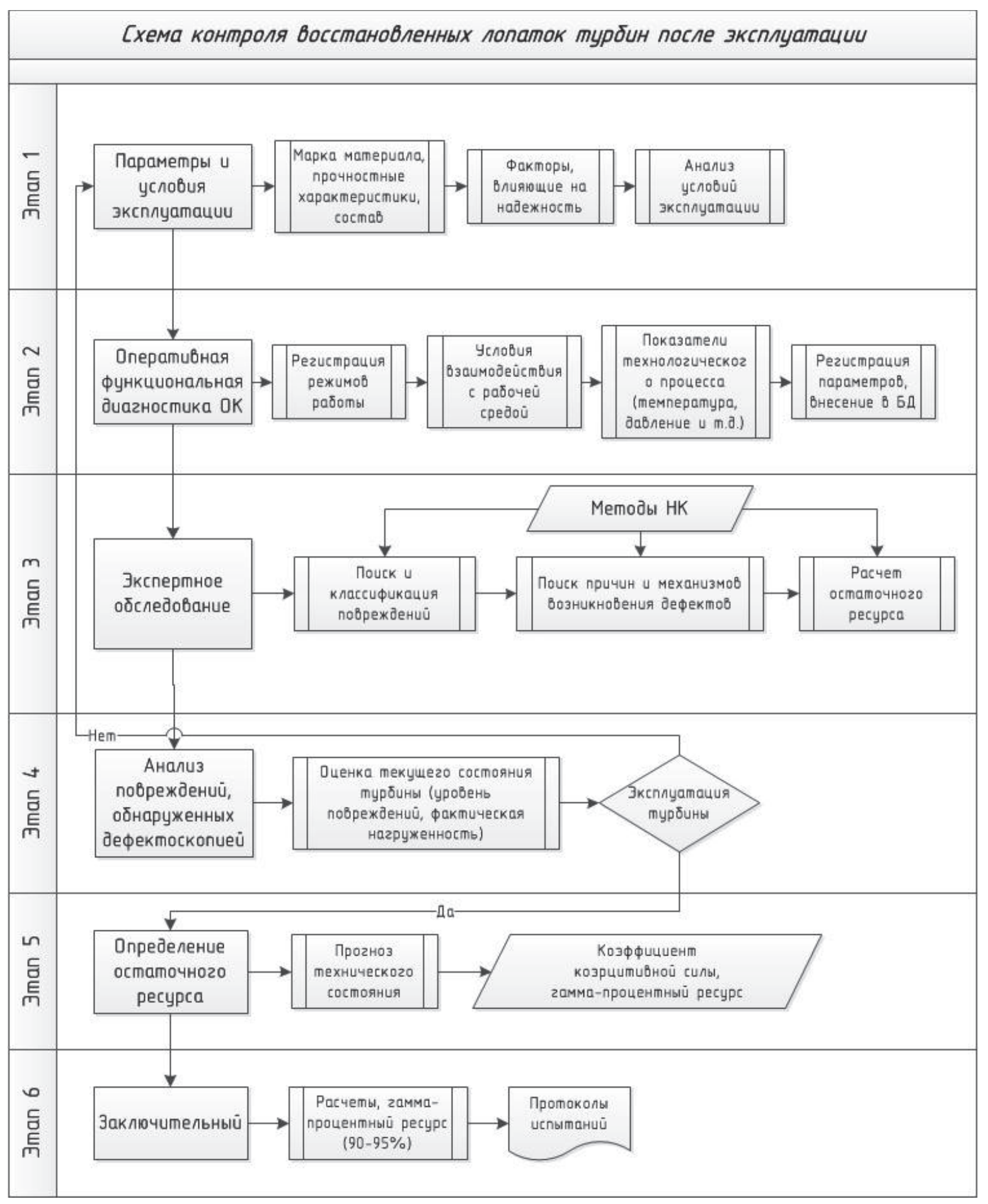

Рис. 1. Схела контроля восстановленных лопаток турбин после эксплуатации

Fig. 1. Scheme for monitoring the recovered turbine blades after operation

Этап № 4. Идентификаиия и интерпретаиия дефектов. Вынесение решения о целесообразности эксплуатации турбины. Кроме этого, проводится оценивание фактической работоспособности турбины и ее динамических режимов нагружения. Результатом проведенной работы является математически-статистическая оценка таких параме- тров, как трещиностойкость, интенсивность развития трещин и их увеличение от размера допустимой глубины $h_{\text {доп }}$ до глубины, предшествующей разрушению $h_{\max }$, интенсивность развития очагов питинга, динамика формирования коэрцитивной силы на неповрежденном участке металла $H_{\text {ст } \max }$ и в зоне повреждений $H_{\text {стах }}$. Полученные количе- 
ственные показатели пограничных значений характеристик турбины в целом и лопаток для прогноза их остаточного ресурса становятся завершающим шагом данного этапа.

Этап № 5. Oпределение остаточного ресурса. Остаточный ресурс лопаток турбин устанавливается многокритериальным подходом путём прогноза безотказной работы по качественным параметрам, характеризующим предельное состояние.

В качестве главного показателя остаточного ресурса рекомендован масштабный коэффициент. Его значение характеризует величину коэрцитивной силы, влияющей на гамма-процентный $\gamma$ ресурс.

Этап № 6. Заключительный. Составляется отчет о фактическом состоянии турбины, предельной наработке $T$ до отказа, определяется локальная зона изменения структуры металла, испытывающая максимальные динамические нагрузки.

\section{Экспериментальные исследования зависимости циклических нагрузок от времени, контроль и измерение усталостных напряжений с адаптацией к производственному процессу}

Исследованиями установлено, что за время эксплуатации оборудования в отдельных деталях создаются локальные очаги усталостных напряжений $(0 У Н)$, которые снижают усталостную и вибрационную прочность восстанавливаемых участков лопаток, работающих в условиях знакопеременных циклических нагрузок $[15,16]$. Основные требования, значения действующих сил и моментов, которые формируют технические и эксплуатационные условия работы турбины, регламентированы ГОСТ 34365-2017 «Турбины тепловые промышленного применения (паровые турбины, газовые турбины со ступенями давления). Общие требования». При оценивании минимальных значений напряжения цикла $\sigma_{\min }$ эксплуатационные параметры (скорость потока пара, скорость вращения турбины, влажность пара, давление, угол атаки пара, температура и др.) обусловливают действующие динамические нагрузки, циклическое изменение которых формирует силы сопротивления, приводящие к нарушению равновесного распределения сил и моментов, влияющих на эффективность и надежность эксплуатации лопаток турбин.

Следовательно, важно конкретизировать технические характеристики паровой турбины T-100/120, на которой установлены восстановленные лопатки (табл. 1). Исследуется турбина типа Т-100/120 номинальной мощностью $110000 \mathrm{kBт}$ при 3000 об/мин. Напряжения изгиба, возникающие под действием газового потока в лопатках, составляют 80-130 МПа.

Зависимости величины циклически действующей нагрузки от временного интервала на практике бывают самыми различными: от структурированных до беспорядочных и мало регламентированных. При экспериментальных исследованиях в качестве показателей цикличности нагрузок выбраны:
- переменные напряжения за один цикл их изменения;

- минимальное напряжение цикла $\sigma_{\min }, \mathrm{H} / \mathrm{m}^{2}$, наименьшее по алгебраическому значению напряжение цикла, равное алгебраической разности среднего напряжения цикла и амплитуды; - максимальное напряжение цикла $\sigma_{\max }, \mathrm{H} / \mathrm{m}^{2}$;

- наибольшее по алгебраическому значению напряжение цикла, равное алгебраической сумме среднего напряжения цикла и амплитуды;

- период цикла $t$ - время одного цикла напряжения в лопатках.

Таблиц 1. Технические характеристики паровой турбины $T-100 / 120$

Table 1. Technical characteristics of the steam turbine T-100/120

\begin{tabular}{|c|c|}
\hline $\begin{array}{l}\text { Технические характеристики } \\
\text { Technical characteristics }\end{array}$ & $\begin{array}{c}\text { Значение } \\
\text { характеристики } \\
\text { Value } \\
\text { of the characteristics }\end{array}$ \\
\hline $\begin{array}{l}\text { Давление (абсолютное) свежего пара, кгс/ } \mathrm{cm}^{2} \\
\text { Fresh steam pressure (absolute), } \mathrm{kgf} / \mathrm{cm}^{2}\end{array}$ & 120 \\
\hline $\begin{array}{l}\text { Tемпература свежего пара, }{ }^{\circ} \mathrm{C} \\
\text { Fresh steam temperature, }{ }^{\circ} \mathrm{C}\end{array}$ & 555 \\
\hline $\begin{array}{l}\text { Частота вращения ротора, об/мин } \\
\text { Rotor speed, r/min }\end{array}$ & 3000 \\
\hline $\begin{array}{l}\text { Номинальная мощность турбины, МВт } \\
\text { Rated power of the turbine, MW }\end{array}$ & 110 \\
\hline $\begin{array}{l}\text { Расход свежего пара при номинальной } \\
\text { мощности, т/ч } \\
\text { Fresh steam consumption at rated power, } \mathrm{t} / \mathrm{h}\end{array}$ & 398 \\
\hline $\begin{array}{l}\text { Hоминальная отопительная нагрузка, Гкал/ч } \\
\text { Nominal heating load, Gcal/h }\end{array}$ & $\begin{array}{c}175 \text { (примерно/ } \\
\text { approximately } 340 \text { ) }\end{array}$ \\
\hline $\begin{array}{l}\text { Максимальная отопительная нагрузка } \\
\text { с учетом использования теплоты пара, } \\
\text { поступающего в конденсатор, для подогрева } \\
\text { сетевой воды, Гкал/ч } \\
\text { Maximum heating load taking into account } \\
\text { the use of steam heat entering the condenser } \\
\text { to heat the mains water, Gcal/h }\end{array}$ & 184 \\
\hline
\end{tabular}

Среднее напряжение цикла определяем по выражению (1): формулы перенабрали, видимо из-за конвертации в более позднюю версию, они стали картинками

$$
\sigma_{m}=\frac{\sigma_{\max }+\sigma_{\min }}{2} ;
$$

амплитуду цикла по формуле (2):

$$
\sigma_{a}=\frac{\sigma_{\max }-\sigma_{\min }}{2}
$$

размах цикла определяем по формуле (3):

$$
\sigma_{a}=\sigma_{\max }-\sigma_{\min } \text {. }
$$

Коэффициент асимметрии цикла определяем по формулам (4), (5)

$$
\begin{gathered}
R=\sigma_{\text {min }} / \sigma_{\text {max }} ; \\
R=\tau_{\text {min }} / \tau_{\text {max }} .
\end{gathered}
$$

Свойство металла противостоять усталости определяется пределом выносливости $\sigma_{R 1}, \mathrm{H} / \mathrm{M}^{2}$ (предел усталости), которое регламентируется значением максимального напряжения цикла с асимметрией $R$, соответствующей заданной долговечно- 


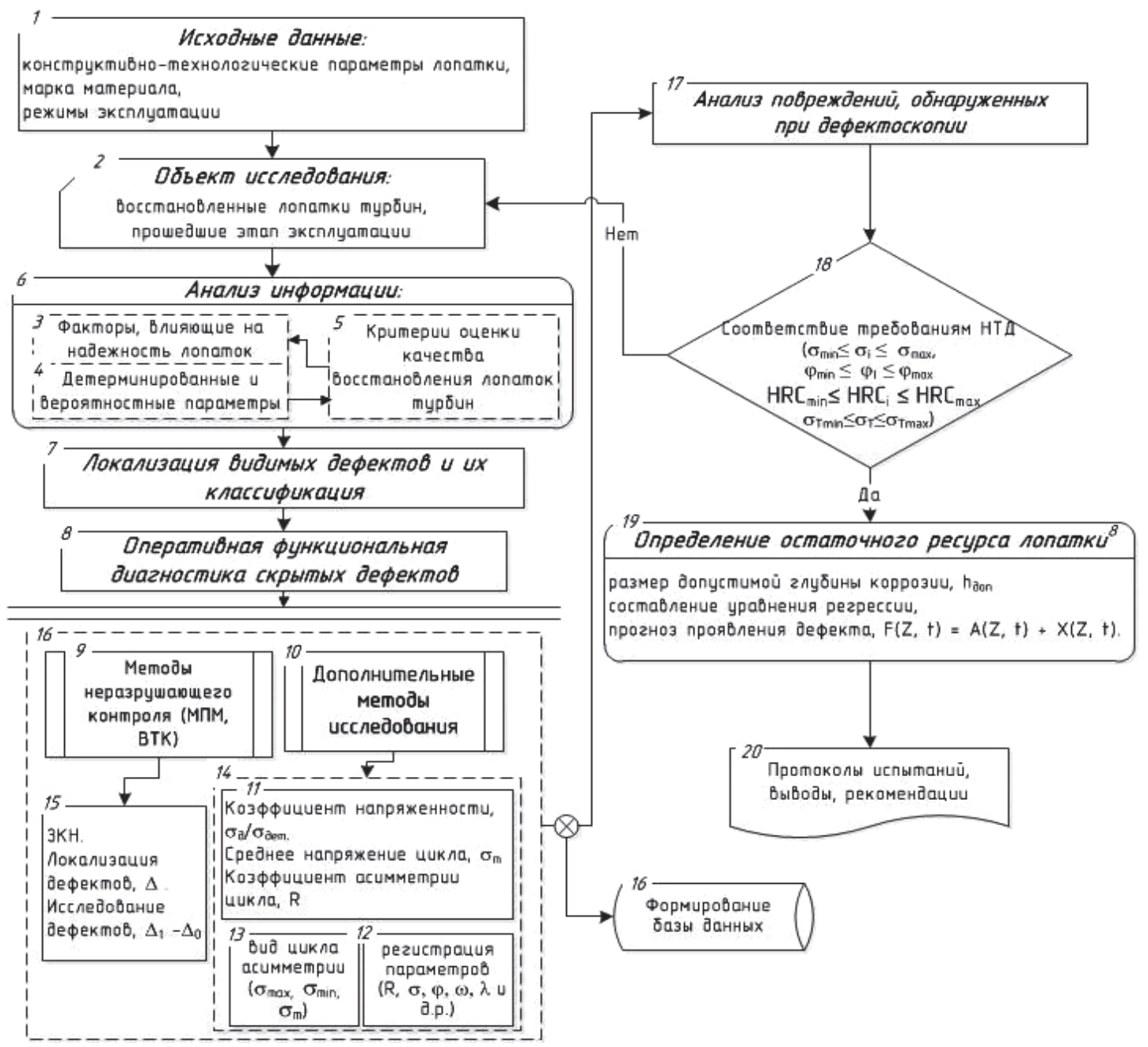

Рис. 2. Блок-схема алгоритма интегрированного метода диагностики лопаток турбин

Fig. 2. Block diagram of the algorithm of integrated turbine blade diagnostics

сти. Предел выносливости при симметричном цикле $\sigma_{-1}$ - это предел выносливости при условии, что $\sigma_{\max }=\sigma_{\min }$, но противоположны по знаку, то есть $R=-1$. В случае, если цикл симметричный, уравнение будет подчиняться синусоидальному закону (6):

$$
\sigma=\sigma_{i}-\sigma_{a} \sin (\omega t+\varphi) \text {. }
$$

Наиболее значимые результаты исследований методов обнаружения и измерения напряженности микроструктуры восстановленных лопаток турбины Т-100/120 реализованы в алгоритме интегрированного метода диагностики (рис. 2).

Разработанный алгоритм позволяет более точно определять зоны формирования напряжений в фазовой структуре лопатки и установить причинноследственную связь зарождения и развития дефектов в прогнозируемый период времени. Для апробации методики были выбраны рабочие лопатки из высокохромистой стали мартенситно-ферритного класса 15Х11МФ, применяемые на ТЭЦ-2 г. Петропавловска. Рассмотрим ключевые этапы алгоритма проведения контроля лопаток турбин.
В процессе оценки соответствия профиля лопаток техническим требованиям осуществлялось измерение остаточных напряжений аттестованным средством измерений марки ИКН-7M-16 (относительная погрешность измеряемого магнитного поля для каждого канала измерений составляет не более $\pm 5 \%$; относительная погрешность измеряемой длины составляет не более $\pm 5 \%$ ), идентификация которых основана на магнитоупругом эффекте и рассеянии магнитного потока в слабых магнитных полях фазовой структуры металла. Этот метод позволил сформировать базу данных причин возникновения фазовых дефектов в структуре нагруженных деталей на стадии предразрушения [17]. Рентгеноструктурный анализ восстановленного образца выполнен на дифрактометре

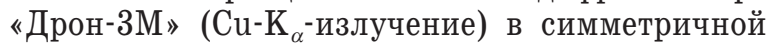
съемке по Брэггу-Брентано.

Предложенный метод не только определяет очаги возможных усталостных дефектов, но также позволяет выявить и интерпретировать ошибки технологического процесса восстановления. Экс- 
периментально доказано, что термодинамические явления наплавочных процессов существенно изменяют коэффициент термического расширения металла восстановленных лопаток [18-21]. Для изделий, функционирующих под влиянием знакопеременных циклических нагрузок и восстановленных плазменным напылением, запас усталостной прочности оценивали следующими параметрами:

- коэффициентом концентрации напряжений:

$$
a_{\sigma}=\sigma_{\max } / \sigma_{H},
$$

где $\sigma_{\max }$ - максимальное напряжение в зоне действия концентрации напряжений, $M H / \mathrm{MM}^{2} ; \sigma_{H}-$ номинальное напряжение в том же сечении, $\mathrm{MH} / \mathrm{MM}^{2}$;

- масштабным фактором распределения:

$$
\varepsilon_{\sigma}=\sigma_{-1 d} / \sigma_{-1},
$$

где $\sigma_{-1 d}$ - предел выносливости образца произвольного диаметра, $\mathrm{MH} / \mathrm{Mv}^{2} ; \sigma_{-1}$ - предел выносливости образцов диаметром 7,5 мм, $\mathrm{MH} / \mathrm{mм}^{2}$.

По результатам испытаний установлено, что зона зарождения и развития дефекта совпадает с плоскостью расчетного сечения. При этом возрастает концентрация внутренних напряжений в точке опасного сечения. При последующем нагружении лопатки концентрация внутренних напряжений в металле возрастает, что сопровождается изменением структурной составляющей. Результаты исследования концентрации внутренних напряжений представлены в виде графика (рис. 3).

Контроль и измерения проводились на длине рабочей кромки лопатки, в местах, наиболее подверженных механическому и эрозионно-динамическому износу. Сканирование шестнадцатиканальным датчиком прибора ИКН-7M-16 проводилось от коренного сечения лопатки к периферийному или, наоборот, по трем осям координат.
Сплошная красная линия на графике (рис. 3) показывает изменение магнитного поля на метр длины образца (А/м), на котором четких всплесков не видно. Красная пунктирная линия показывает допустимое значение напряжений для данного вида изделия. Синяя сплошная линия графика (градиента) показывает места всплеска напряженности магнитного поля, характеризующие формирование концентраций напряжений на каждом миллиметре образца (А/мм). Изменение знака показывает направление изогнутости.

\section{Анализ данных, полученных}

\section{в результате экспериментальных исследований}

По итогам контроля определены участки лопатки, работающие в наиболее напряженных условиях. Это участки, у которых линии концентраций напряжений и деформации расположены поперечно и имеют максимальный коэффициент интенсивности напряжений. Значения этого коэффициента рассчитываются автоматически и отображаются на экране по вызову мастера-диагноста.

Локальные очаги напряженности структуры формируются в местах возникновения дефектов образца на контрольной длине от 71 до 82 мм и на 10 мм от критической точки (рис. 3). Если в опасном сечении, при выбранной схеме защемления, изгибающий момент и действующие напряжения имеют наибольшее значение, то при изгибе других видов эти величины меньше примерно на 20-25\% .

Значения остаточных напряжений в зоне восстановления не превышали пороговые значения. Структура металла при этом представляет сорбит, ориентированный на мартенсит, и обеспечивает микротвердость восстановленной лопатки от 544 до $590 \mathrm{HV}$. Как известно, твердость и микротвердость являются одними из качественных критериев процесса плазменного восстановления. Изме-

\section{О6разец №4}

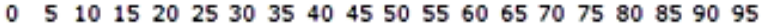

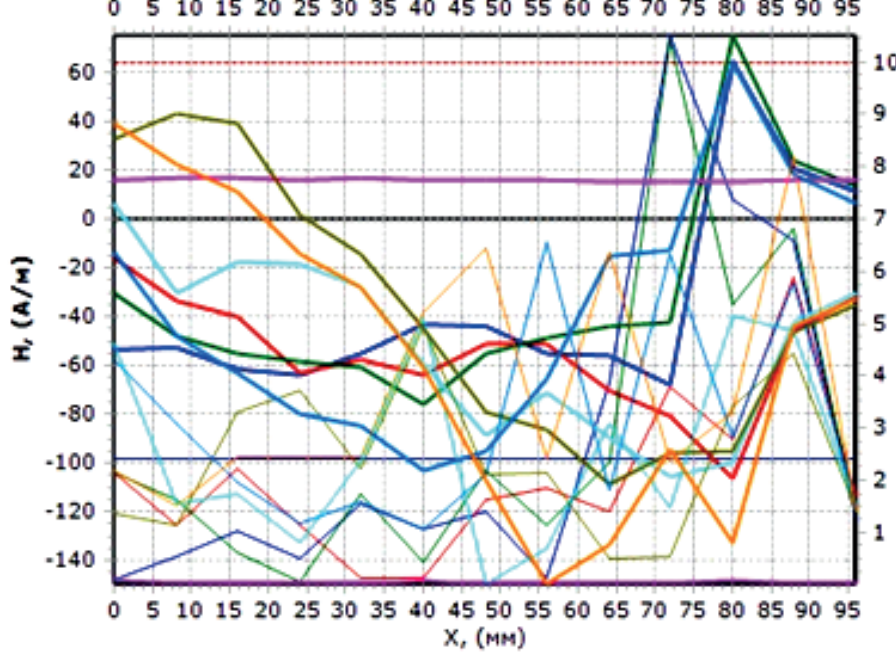

Рис. 3. График зон концентрации напряжений в лопатки турбины

Fig. 3. Graph of stress concentration zones in turbine blades

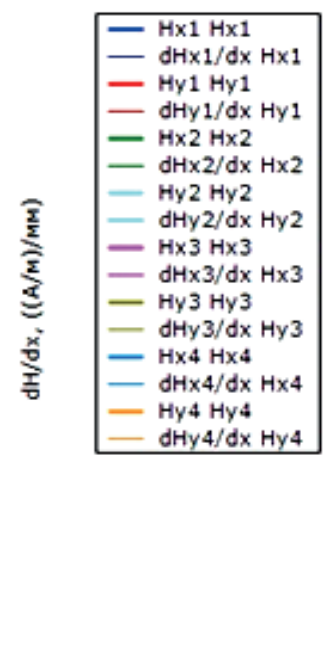


рение микротвердости проводилось на микротвердомере ПМТ-3 с нагрузкой по ГОСТ 9450-76 «Измерение микротвердости вдавливанием алмазных наконечников» от основы поверхности образца до края поверхности покрытия к основе с захватом переходной зоны.

Структура в виде сорбита, ориентированного на мартенсит, образуется при определенном химическом составе материала и температурном режиме воздействия. Данные условия являются ключевыми параметрами технологического процесса плазменного восстановления и достигаются при разных режимах наплавки, напыления или при «отпуске». В технологическом процессе восстановления лопаток присутствует операция «отпуск» переходной зоны от наплавленного металла к основному, вследствие чего формируется ферритная прослойка. Это происходит в результате вынужденного диффундирования углерода. Значит, структурные дефекты детали являются следствием неэффективно выбранных режимов восстановления.

Следовательно, обоснована необходимость в установлении зависимости между изменениями внутренних усталостных напряжений, структурно-фазового состояния металла, предшествующими появлению микротрещин и полному разрушению, и технологическими параметрами восстановления лопаток турбины ТЭЦ и ГЭС.
По результатам исследования усталостных напряжений в структуре металла лопатки авторами впервые сформирована база данных, позволяющая интерпретировать рассеяние магнитного потока в слабых магнитных полях на единицу площади, в зависимости от фазовой структуры лопаток и особенностей технологии их восстановления. Результаты исследования причин напряженного состояния структуры металла позволили сформировать базу данных по соответствию структурно-фазового состава напряженности магнитного поля и дефекта (табл. 2).

Каждому виду дефекта соответствует определенная структурно-фазовая составляющая и уровень напряженности магнитного поля.

Например, при мелкозернистом перлите и ферритных включениях очень слабо прослеживается граница первичных аустенитных зерен (образец № 4), что приводит к микротрещинам и обеспечивает высокую пористость материала при термообработке. Установленная причинно-следственная связь дефекта и напряженности магнитного поля характеризует неэффективные параметры режимов восстановления, повлекшие слабую диффузию углерода из основного металла и недостаточную величину погонной энергии при плазменном напылении.

Рассматривая образец № 1 можно отметить, что всплеск напряженности магнитного поля по

Таблица 2. Результаты исследования причин напряженного состояния структуры металла лопатки турбины

Table 2. Results of investigation of the causes of the stressed state of the turbine blade metal structure

\begin{tabular}{|c|c|c|c|c|c|c|}
\hline 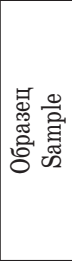 & 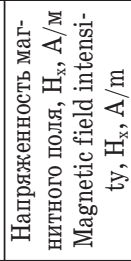 & $\begin{array}{l}\text { Структурно-фазовое состояние } \\
\text { Structural-phase state }\end{array}$ & 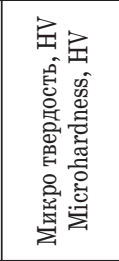 & $\begin{array}{l}\text { Вид обработки } \\
\text { Kind } \\
\text { of processing }\end{array}$ & $\begin{array}{l}\text { Дефект } \\
\text { Defect }\end{array}$ & $\begin{array}{l}\text { Причина дефекта } \\
\text { Cause of the defect }\end{array}$ \\
\hline 1 & $62 \pm 1,76$ & $\begin{array}{l}\text { Сорбит, ориентированный на мартен- } \\
\text { сит } \\
\text { Martensite - oriented sorbitol }\end{array}$ & $544-590$ & $\begin{array}{l}\text { Оплавление } \\
\text { без присадки } \\
\text { Reflow without } \\
\text { additive }\end{array}$ & $\begin{array}{l}\text { Изменение проектной } \\
\text { геометрии (деформация) } \\
\text { Changing the design geo- } \\
\text { metry (deformation) }\end{array}$ & $\begin{array}{l}\text { Малая глубина закалки, не- } \\
\text { правильные режимы эксплуа } \\
\text { тации турбины } \\
\text { Shallow depth of hardening, } \\
\text { wrong modes of turbine opera- } \\
\text { tion }\end{array}$ \\
\hline 2 & $54 \pm 1,42$ & $\begin{array}{l}\text { Средне игольчатый мартенсит и ле- } \\
\text { гированный феррит, выделившейся } \\
\text { по границам первичных аустенит- } \\
\text { ных зерен в период кристаллизации } \\
\text { Medium-needled martensite and doped } \\
\text { ferrite precipitated along the bounda- } \\
\text { ries of the primary austenitic grains } \\
\text { during the crystallization period }\end{array}$ & $746-1200$ & $\begin{array}{l}\text { Плазменное } \\
\text { напыление } \\
\text { Plasma spraying }\end{array}$ & - & - \\
\hline 3 & $76 \pm 1,82$ & $\begin{array}{l}\text { Грубая игольчатая структура. } \\
\text { Эвтектический ледебурит } \\
\text { Rough-needled structure. } \\
\text { Eutectic ledeburite } \\
\end{array}$ & $446-458$ & $\begin{array}{l}\text { Плазменное } \\
\text { напыление } \\
\text { Plasma spraying }\end{array}$ & $\begin{array}{l}\text { Хрупкость } \\
\text { Fragility }\end{array}$ & $\begin{array}{l}\text { Перегрев металла } \\
\text { в процессе напыления } \\
\text { Metal overheating during pla- } \\
\text { sma spraying }\end{array}$ \\
\hline 4 & $80 \pm 2,4$ & $\begin{array}{l}\text { Мелкозернистый перлит + немного } \\
\text { феррита. Очень слабо прослеживает- } \\
\text { ся границы первичных аустенитных } \\
\text { зерен } \\
\text { Fine-grained perlite + some ferrite. } \\
\text { The boundaries of primary austenitic } \\
\text { grains are very poorly traced }\end{array}$ & $302-318$ & $\begin{array}{l}\text { Плазменное } \\
\text { напыление } \\
\text { Plasma spraying }\end{array}$ & $\begin{array}{l}\text { Трещины и единичные } \\
\text { поры } \\
\text { Cracks and single pores }\end{array}$ & $\begin{array}{l}\text { Слабая диффузия углерода из } \\
\text { основного металла - недоста- } \\
\text { точная величина погоной } \\
\text { энергии при напылении } \\
\text { Weak diffusion of carbon from } \\
\text { the base metal - insufficient } \\
\text { amount of running energy du- } \\
\text { ring spraying }\end{array}$ \\
\hline
\end{tabular}


результатам измерений составил $\mathrm{H}_{\mathrm{x}}=62 \pm 1,76 \mathrm{~A} / \mathrm{m}$ при доверительной вероятности $95 \%$. Это соответствует структурно-фазовому состоянию сорбита, ориентированного на мартенсит, и характеризуется отсутствием технологических дефектов. Однако при тяжелых режимах эксплуатации турбины возрастают динамические и вибрационные нагрузки, при которых в лопатке развиваются усталостные процессы и приводят ее к деформации и разрушению. При формировании фазовой структуры металла в виде средне игольчатого мартенсита и легированного феррита, выделившегося по границам первичных аустенитных зерен, дефекты отсутствуют, микротвердость составляет 746-1200 HV, а напряженность магнитного поля составляет $\mathrm{H}_{\mathrm{x}}=54 \pm 1,42 \mathrm{~A} / \mathrm{м}$. При значениях напряженности $\mathrm{H}_{\mathrm{x}}=76 \pm 1,82-80 \pm 2,4 \mathrm{~A} / \mathrm{м}$ магнитного поля фазовая структура металла имеет иные составляющие (мелкозернистый перлит), приводящие к дефектам, усталостным напряжениям и разрушению, именно поэтому данные условия являются критическими.

Таким образом, сформированная база данных концентраций напряжений лопатки в зависимости от структуры металла позволяет установить причинно-следственные связи между дефектом, технологическими режимами восстановления и режимами эксплуатации агрегата.

Изменение ресурса лопаток во многом определяется структурно-фазовыми составляющими, а степень наработки на отказ в большинстве случаев зависит от зарождения усталостных трещин, первостепенная причина которых в концентрации внутренних напряжений. Следовательно, доказана необходимость в пересмотре методики диагностики и прогнозирования ресурса, а также адекватность результатов предложенного комплексного метода оценки ресурсной долговечности оборудования.

Основным показателем ресурса должно быть не количество рабочих циклов $N_{H E}$ до полного разрушения лопатки, как предписано в классических методиках и справочниках [21], а количество циклов до появления предельных внутренних напряжений, предшествующих образованию микротрещин. В свою очередь, представленная поэтапная интегрированная методика прогнозирования должна завершаться расчетом коэффициента трещиностойкости с учетом коэрцитивной силы.

\section{Заключение}

В результате проведенных исследований влияния концентрации усталостных напряжений на формирование фазовой структуры лопаток турбины ТЭЦ установлено, что научно-теоретические положения теории распределения потоков остаточной намагниченности в деградированной микроструктуре материала полностью применимы для анализа временных характеристик вибрационной надежности, случайных нагрузок. Практические и экспериментальные результаты иссле- дований позволили разработать эффективный метод интегрированной оценки усталостных напряжений в структуре восстановленных лопаток турбин ТЭЦ и обосновать его применимость и адекватность измеренных параметров напряженности.

Предлагаемая методика позволяет своевременно определять лопатки турбин, которые работают с зонами КН от $76 \pm 1,82$ до $80 \pm 2,4 \mathrm{~A} /$ м в перлитной фазе, т. е. на критической стадии пред разрушения зерен структуры. При формировании фазовой структуры металла в виде средне игольчатого мартенсита и легированного феррита, выделившегося по границам первичных аустенитных зерен, дефекты отсутствуют, микротвердость составляет 746-1200 HV, а напряженность магнитного поля $\mathrm{H}_{\mathrm{x}}=54 \pm 1,42 \mathrm{~A} / \mathrm{м}$. Значение $\mathrm{H}_{\mathrm{x}}=54 \pm 1,42 \mathrm{~A} / \mathrm{м}$ оптимальное для безотказной работы лопаток, имеющих высокий ресурсный запас $10^{6}$ прочности. Разработан алгоритм проведения диагностики лопаток с использованием методов неразрушающего контроля, который синтезирует взаимодействие метрологического оборудования и методической составляющей применения математического аппарата, что повышает точность оценки остаточного ресурса лопаток и турбины в целом.

Производственные испытания предложенного интегрированного метода позволят безошибочно определять зоны зарождения очагов внутренних напряжений лопаток на ранней стадии их формирования. Сформированная база данных о дефекте позволяет анализировать структурную и фазовую составляющую лопатки как до восстановления, так и после. Предложенный подход комплексной оценки изменения структурной составляющей позволит расширить область понимания формирования усталостных напряжений, оценить возможные критические нагрузки при эксплуатации и сформировать критерии для разработки энергосберегающей технологии восстановления высококонцентрированными источниками плазменной энергии.

Комплексное использование методов неразрушающего контроля и индекса технического состояния (интегрированным методом) для исследования динамики и прогнозирования изменения свойств лопатки турбины позволяет обеспечить абсолютную информационную помощь принятия конструктивно-технологических решений, направленных на снижение количества отказов оборудования и предотвращения техногенных катастроф с участием энергетических комплексов. На этом принципе будет реализовано планирование энергоэффективных работ и выбор стратегии повышения надёжности производства и преобразования энергии технологическими и эксплуатационными методами. Системный подход и точное прогнозирование комплекса критериальных показателей позволяют значительно увеличить ресурс работы энергетического оборудования и существенно снизить себестоимость производства электрической и тепловой энергии за счет преобразования энергии георесурсов. 
Решена научная проблема по созданию единой физической модели и определению границ применимости данного метода, что повышает достоверность и однозначность получаемых результатов по оценки ресурса лопаток турбин ТЭЦ.

Результаты исследований позволили определить четкие границы условий надежной эксплуатации турбины ТЭЦ. Установлено, что безопасный режим эксплуатации энергетического оборудования должен находиться в пределах $\mathrm{H}_{\mathrm{x}}=62 \pm$ $\pm 1,76-76 \pm 1,82$ А/м. Данные значения возможно достичь при формировании фазовой структуры материала, приближенной или сходной со среднеигольчатым мартенситом и легированным ферритом. Следовательно, предложенная методика позволяет не точечно прогнозировать вибрационный ресурс энергетического комплекса, но и совершенствовать технологию восстановления изношенных частей. Данная задача решается за счет регулирования исследованных термодинамических параметров и установленных закономерностей изменения причинно следственных связей между технологией и

\section{СПИСОК ЛИТЕРАТУРЫ}

1. Проблемы при эксплуатации лопаток последних ступеней паровых турбин после восстановительного ремонта / А.М. Филиппов, Е.О. Голикова, А.Н. Сидоров, А.С. Азин // Актуальные проблемы в машиностроении. - 2016. - № 3. - С. 17-22.

2. Должанский П.Р., Егоров А.В., Доброхотов С.Э. Повышение работоспособности рабочих лопаток последних ступеней турбин Т-250 // Металл оборудования ТЭС. Проблемы и перспективы» ОАО «ВТИ»: Сборник докладов научно-практической конференции. - М., 2006. - С. 79-82.

3. Должанский П.Р., Доброхотов С Э. Повышение эксплуатационной надёжности рабочих лопаток последних ступеней турбин Т-250/300-240 // Надёжность и безопасность энергетики. - 2008. - № 1. - С. 56-59.

4. Abhijit R. Take Stock of Turbine Blades Failure Phenomenon // Journal of the Institution of Engineers (India): Series C. 2018. - V. 99. - Iss. 1. - P. 97-103.

5. Савинкин В.В., Ратушная Т.Ю., Абильмажинова А.А. Исследования концентрации внутренних напряжений в лопатках турбины ТЭЦ методом магнитной памяти металла // Научнотехнический журнал «Метрология». - 2017. - № 1. - С. 33-42.

6. Ali Sophian, Guiyun Tian, Mengbao F. Pulsed Eddy Current Nondestructive Testing and Evaluation: a Review // Chinese Journal of Mechanical Engineering. - 2017. - V. 30. - Iss. 6. P. 1473-1474.

7. Дубов А.А. Диагностика турбинного оборудования с использованием магнитной памяти металла. - М.: ЗАО «ТИССО», 2009. -148 c.

8. Coupling method of magnetic memory and eddy current nondestructive testing for retired crankshafts / Chen Ni, Lin Hua, Xiaokai Wang, Zhou Wang, Xunpeng Qin, Zhou Fang // Journal of Mechanical Science and Technology. - 2016. - V. 30. - Iss. 7. P. 3097-3104.

9. 0 проблемах применимости метода магнитной памяти металла при контроле напряженно-деформированного состояния металлоконструкций / М.Б. Аркулис, М.П. Барышников, Н.И. Мишенева, Ю.И. Савченко // Дефектоскопия. - 2009. № $8 .-$ C. $10-12$. формированием фазовой структуры, обеспечивающей запас прочности к динамическим нагрузкам.

Научные исследования проведены при совместном партнерском сотрудничестве с производственной средой машиностроения ремонтно-восстановительной отрасли ТОО «Ремплазма», ТОО «Завод многопрофильного оборудования» и АО «СЕВКАЗЭНЕРГО» ТЭЦ-2 г. Петропавловск.

Металлографические исследования восстановленных лопаток проведены на базе и при поддержке Физико-технического института Национальной Академии Наук Беларуси г. Минск.

Установленные зависимости ОУН от микроструктуры материала определены в ходе грантовых исследований, финансируемых МОН РК по проекту № 2607/ГФ4-15-ОТ «Разработка и внедрение энергоэффективной технологии восстановления лопаток сложной геометрии паровых и газовых турбин ТЭЦ высококонцентрированным источником плазменной энергии с адаптивной системой управления процессами» договор № 63 от 27.02.2017 года.

10. An algorithm for evaluating the state of a generating unit based on the identification measurements of vibrosignals / Yu.N. Klikushin, K.T. Koshekov, V.Yu. Kobenko, E.S. Trunin // Russian Journal of Nondestructive Testing. - 2014. - V. 50. - Iss. 7. P. 413-418.

11. Zhang W.W., Cong S. Process optimization and performance evaluation on laser beam welding of austenitic/martensitic dissimilar materials // The International Journal of Advanced Manufacturing Technology. - 2017. - V. 92. - Iss. 9-12. - P. 4161-4168.

12. Murakami R., Akizono K. A study on the impact fatigue crack propagation into the welding residual stresses field // International Journal of Fracture. - 1983. - V. 23. - Iss. 4. P. R151-R154.

13. Artamonov V.V., Artamonov V.P. Diagnostics of the causes of the operational destruction of rotor blades of gas turbines // Russian Journal of Nondestructive Testing. - 2013. - V. 49. Iss. 9. - P. 538-542.

14. Corrosion Fatigue of Rotating Blades / M.A. Virchenko, E.V. Levchenko, B.A. Arkad'ev, Yu.F. Kosyak, V.P. Sukhinin, Yu.Ya. Kachuriner // Thermal Engineering. - 1997. - V. 44. № 6. - P. 464-468.

15. Савинкин В.В., Ратушная Т.Ю. Математическое описание основных факторов, повышения газодинамической нагрузки турбины ТЭЦ // Вестник, Серия техническая, СКГУ им. М. Козыбаева. - 2016. - № 4 (33). - С. 114-118.

16. Расчеты на прочность в машиностроении: Справочник в 5 т. / под ред. С.Д. Пономарев, В.Л. Бидерман. - М.: Машиздат., 1956-1958. - 1118 c.

17. Неразрушающий контроль: Справочник в 7 т. / под общ. ред. В.В. Клюева. Т. 7. В 2 кн. Кн. 1: Вибродиагностика. - М.: Машиностроение, 2005. -829 c.

18. Загидулин Р.В., Загидулин Т.Р. Исследование зависимости поля остаточной намагниченности от напряженно-деформированного состояния металла стального изделия. Ч. 2. Зависимость поля остаточной намагниченности металла от механического напряжения // Контроль. Диагностика. - 2011. № 8 (158). - C. 14-20.

19. Новиков В.Ф., Семенов В.В., Бахарев М.С. Возможности определения предела пропорциональности (предела микротеку- 
чести) стали по кривым магнитоупругого размагничивания // Контроль. Диагностика. - 2006. - № 4. - С. 16-17.

20. Blachnio J., Bogman M. A Non-destructive method to assess condition of gas turbine blades, based on the analysis of blade-surface images // Russian Journal of Nondestructive Testing. 2010. - № 11. - P. 89-98.
21. Artamonov V.V., Artamonov V.P. Diagnostics of the causes of the operational destruction of rotor blades of gas turbines // Russian Journal of Nondestructive Testing. - 2013. - T. 49. - № 9. P. 538-542.

\section{Информация об авторах}

Савинкин В.В., доктор технических наук, доцент, заведующий кафедрой транспорта и машиностроения, Северо-Казахстанский государственный университет им. М. Козыбаева.

Кузнецова В.Н., доктор технических наук, профессор, декан факультета «Нефтегазовая и строительная техника», Сибирский государственный автомобильно-дорожный университет.

Ратушная Т.Ю., магистр, докторант кафедры транспорта и машиностроения, Северо-Казахстанский государственный университет им. М. Козыбаева.

Киселев Л.А., заслуженный изобретатель КазССР, директор ТОО «Ремплазма». 


\title{
METHOD OF INTEGRATED ASSESSMENT OF FATIGUE STRESSES IN THE STRUCTURE OF THE RESTORED BLADES OF CHP AND HPS
}

\author{
Vitalii V. Savinkin', \\ cavinkin7@mail.ru
}

Viktoria N. Kuznetsova²,
dissovetsibadi@bk.ru

Tatyana Yu. Ratushnaya', tratushnaia@mail.ru

\author{
Leonid A. Kiselev ${ }^{3}$, \\ remplazma@mail.ru \\ ${ }^{1}$ North Kazakhstan State University named after M. Kozybaev, \\ 86, Pushkin Street, Petropavlovsk, 150000, Republic of Kazakhstan. \\ 2 Siberian State Automobile and Highway University, \\ 5, Mira Avenue, Omsk, 644080, Russia. \\ ${ }^{3}$ LLP «Remplazma», \\ 22, Ya. Gashek Street, Petropavlovsk, 150000, Republic of Kazakhstan.
}

The relevance of research is caused by the necessity to develop the method of precise definition of the resource durability and reliability of heavily loaded equipment used for energy production and conversion on the basis of geo-resources (hydro, CHP), based on identification of zones of concentration of internal stresses, as an indicator of phase change in the material structure of the parts.

The main aim of the research is to increase resource durability of loaded turbine blades of hydro and thermal power stations due to implementation of the developed integrated methodology of precise prediction of structural-phase changes at the early stages of fatigue defects.

The object of research is the turbine blades of CHP and HPP, restored by a source of laser-plasma energy.

The complex research method was used to study the concentration of internal stresses in the structure of loaded parts subjected to dynamic loads. The methodology of system analysis was used, as well as the method of distribution of the residual magnetization field in the degraded microstructure of the material. In development of the algorithm of the integrated method of diagnosis and accurate prediction, the methods of mathematical statistics were used. The restored parts were studied by nondestructive testing followed by analysis and processing of the results.

Result. The authors have developed the algorithm of diagnostics of the restored details of high-tech complexes of energy production and transformation of georesources, on the example of turbine blades of hydroelectric power station. A database of blade stress concentrations depending on the phase structure of the metal was formed, which allows establishing the cause-and-effect relationships between the defect, technological recovery modes and operating modes of the unit. The scientific problem of creating a single physical model is solved and the limits of applicability of this method are determined. The authors determined the safe operation mode of power equipment $\left(H_{x}=62-76 \mathrm{~A} / \mathrm{m}\right)$, which increases the turbine life by 1,7 times.

\section{Key words:}

Fatigue stresses, phase structure, turbine blade, integrated assessment, non-destructive testing methods.

\section{REFERENCES}

1. Filippov A.M., Golikova E.O., Sidorov A.N., Azin A.S. Problems in the operation of blades of the last stages of steam turbines after recovery repair. Actualnye problemy $v$ mfshinostroenii, 2016, no. 3, pp. 17-22. In Rus.

2. Dolzhansky P.R., Egorov A.V., Dobrokhotov S.E. Povyshenie rabotosposobnosti rabochikh lopatok poslednikh stupeney turbin T-250 [Increase of working capacity of working blades of last stages of turbines T-250]. Metall oborudovanya TES. Problemy i perspektyvy OAO «VTI». Sbornik dokladov nauchno-prakticheskoy konferentsii [Collection of reports of scientifically-practical conference. Metal of the equipment of CHP. Problems and prospects]. Moscow, 2006. pp. 79-82.

3. Dolzhansky P.R., Dobrokhotov S.E. Increase of operational reliability of working blades of last stages of turbines T-250/300-240. Reliability and safety of power, 2008, no. 1, pp. 56-59. In Rus.
4. Abhijit R. Take Stock of Turbine Blades Failure Phenomenon. Journal of the Institution of Engineers (India): Series C, 2018, vol. 99, Iss. 1, pp. 97-103.

5. Savinkin V.V., Ratushnaya T.Yu., Abilmazhinova A.A. Issledovaniya kontsentratsii vnutrennikh napryazheny v lopatkakh turbiny TETs metodom magnitnoy pamyati metalla [Investigations of concentration of internal stresses in turbine blades of CHP by the method of magnetic metal memory]. Scientific and technical journal «Metrology», 2017, no. 1, pp. 33-42. In Rus.

6. Ali Sophian, Guiyun Tian, Mengbao F. Pulsed Eddy Current Nondestructive Testing and Evaluation: A Review. Chinese Journal of Mechanical Engineering, 2017, vol. 30, Iss. 6, pp. 1473-1474.

7. Dubov A.A. Diagnostyka turbinnogo oborudovaniya s ispolzovaniem magnitnoiy pamyaty metalla [Diagnostics of turbine equipment using magnetic memory of metal]. Moscow, 2009. 148 p.

8. Chen Ni, Lin Hua, Xiaokai Wang, Zhou Wang, Xunpeng Qin, Zhou Fang. Coupling method of magnetic memory and eddy cur- 
rent nondestructive testing for retired crankshafts. Journal of Mechanical Science and Technology, 2016, vol. 30, Iss. 7, pp. 3097-3104.

9. Arkulis M.B., Baryshnikov M.P., Misheneva N.I., Savchenko Yu.I. O problemakh primenymosty metoda magnitnoy pamyati metalla pri kontrole napryazhenno-deformirovannogo sostoyaniya metallokonstruktsy [0n the problems of the magnetic metal memory method applicability in controlling the stress-strain state of metal structures]. Defectoscopy, 2009, no. 8. pp. 10-12.

10. Klikushin Yu.N., Koshekov K.T., Kobenko V.Yu., Trunin E.S. An algorithm for evaluating the state of a vibrosignals. Russian Journal of Nondestructive Testing, 2014, vol. 50, Iss. 7, pp. $413-418$.

11. Zhang W.W., Cong S. Process optimization and performance evaluation on laser beam welding of austenitic/martensitic dissimilar materials. The International Journal of Advanced Manufacturing Technology, 2017, vol. 92, Iss. 9-12, pp. 4161-4168.

12. Murakami R., Akizono K. A study on the impact of fatigue crack propagation in the course of the study of isolated stresses field. International Journal of Fracture, 1983, vol. 23, Iss. 4, pp. R151-R154.

13. Artamonov V.V., Artamonov V.P. Diagnostics of the causes of the operational destruction of rotor blades of gas turbines. Russian Journal of Nondestructive Testing, 2013, vol. 49, Iss. 9, pp. $538-542$.

14. Virchenko M.A., Levchenko E.V., Arkad'ev B.A., Kosyak Yu.F., Sukhinin V.P., Kachuriner Yu.Ya. Corrosion Fatigue of Rotating Blades. Thermal Engineering, 1997, vol. 44, no. 6, pp. 464-468.

15. Savinkin V.V., Ratushnaya T.Yu. Matematichescoe opisanie osnounykh faktorou povysheniya gazodinamicheskoy nagruzki tur- biny TEC [Mathematical description of the main factors for increasing the gas-dynamic load of the turbine of CHP]. Vestnik. Seria tekhnicheskaya, SKGU im. M. Kozybaeva, 2016, no. 4 (33), pp. 114-118.

16. Raschety na prochnost $v$ mashinostroenii: Spravochnik $v 5$ tomakh [Calculations for strength in mechanical engineering: a handbook in 5 volumes]. Ed. by S.D. Ponomarev. Moscow, 1956-1958. $1118 \mathrm{p}$.

17. Nerazrushaushchy kontrol. Spravochnik. $V 7$ tomakh. [Non-destructive testing: Handbook. In 7 volums]. Ed. by V.V. Kluev, Moscow, Mechanical engineering Publ., 2005. 829 p.

18. Zagidulin R.V., Zagidulin T.R. Research of the dependence of the residual magnetization field on the stress-strain state of the metal of the steel product. P. 2. Dependence of the residual magnetization field of a metal on the mechanical stress. Control. Diagnostics, 2011, no. 8 (158), pp. 14-20. In Rus.

19. Novikov V.F., Semenov V.V., Bakharev M.S. The possibilities for determining the limit of proportionality (the limit of microtemperature) of steel along the curves of magnetoelastic demagnetization. Control. Diagnostics, 2006, no. 4, pp. 16-17. In Rus.

20. Blachnio J., Bogman M. A Non-destructive method for assessing the condition of gas turbine blades, based on the analysis of bladesurface images. Russian Journal of Nondestructive Testing, 2010, no. 11, pp. 89-98.

21. Artamonov V.V., Artamonov V.P. Diagnostics of the causes of the operational destruction of rotor blades of gas turbines. Russian Journal of Nondestructive Testing, 2013, vol. 49, no. 9, pp. 538-542.

\section{Information about the authors}

Vitalii V. Savinkin, Dr. Sc., associate professor, head of the department, North Kazakhstan State University named after M. Kozybaev.

Victoria N. Kyznetsova, Dr. Sc., professor, Siberian State Automobile and Highway University.

Tatyana Yu. Ratushnaya, postgraduate student, North Kazakhstan State University named after M. Kozybaev.

Leonid A. Kiselev, Honored Inventor of the KazakhSSR, Director of the LLP «Remplazma». 